\title{
Component fractionation of wood-tar by column chromatography with the packing material of silica gel
}

\author{
WANG Ze, LIN WeiGang, SONG WenLi ${ }^{*}$, DU Lin, LI ZhengJie \& YAO JianZhong \\ State Key Laboratory of Multi-Phase Complex System, Institute of Process Engineering, Chinese Academy of Sciences, Beijing 100190, China
}

Received October 31, 2009; accepted July 28, 2010

\begin{abstract}
Bio-oil can be an important fuel resource for automobiles in the future, while its complex composition restricts the direct application of the bio-oil extremely. So it is necessary to separate the complex mixture to relatively simplified fractions for goal directed specific treatments to reach the fuel quality for automobiles, and meanwhile different functional chemical materials and fine chemicals can be obtained. So it is significant to investigate the bio-oil component separating methods. Herein the method of column chromatography by the packing material of silica gel with two series of eluants of cyclohexane-benzene-methanol and cyclohexane-dichloromethane-methanol were investigated for component fractionation of the raw wood tar (oil fraction of the liquid product by slow pyrolysis of wood). The analytical results show that the components in cyclohexane are rich in alkoxyl-monophenols; the components of alkyl-monophenols and five ring oxygen-containing compounds are abundant in benzene and in dichloromethane similarly; in the methanol fraction, the components are diverse and diphenols are relatively in higher content, comparatively small polar molecules and five ring oxygen-containing compounds are more abundant in the methanol fraction after being eluted by dichloromethane, and the content of 1-(4-hydroxy-3-methoxyphenyl) -2-propanone is higher after being eluted by benzene.
\end{abstract}

biomass, wood-tar, column chromatography

Citation: Wang Z, Lin W G, Song W L, et al. Component fractionation of wood-tar by column chromatography with the packing material of silica gel. Chinese Sci Bull, 2011, 56: 1434-1441, doi: 10.1007/s11434-010-4144-x

With declining petroleum resource and more concerns on environment and climate, the development for renewable energy is getting more necessary. Biomass is an important renewable and environment-benign energy resource, but in low energy density, so it is advantageous to convert biomass to the pyrolytic liquid product of bio-oil $[1,2]$. Bio-oil can be directly used for burning in boilers, and also can be a potential resource for fuel of automobiles. While the components in bio-oil are very complex with high oxygen content, and therefore the method of component fractionation is considered for specific refinery for fuel and for chemicals. So it is significant to study the methods for component separation. Presently the main separating methods are solvent extraction, distillation, and column chromatography.

Through solvent extraction, neutral compounds, phenols,

*Corresponding author (email: wlsong@ home.ipe.ac.cn) acids etc. can be obtained, and some frequently used solvents are hexane, benzene, toluene, ether, ethyl acetate, chloroform, methanol, ethanol etc. [3-6]. As in petrochemical industry, distillation is also a common method in bio-oil fractionation [7-12], while the effect was not very satisfactory due to the conversion of much heat sensitive components in bio-oil by pyrolysis and polymerization reactions. To solve the problem, some modified distillation like flash distillation, vacuum distillation, and molecular distillation are also tested, combined with solvent extraction, and the effect can be improved.

Compared with solvent extraction and distillation, the reports on column chromatographic separation of bio-oil are much less. In Ba's work [13], an softwood bark bio-oil was eluted by solvents of pentane, benzene, dichloromethane, ethyl acetate, and methanol in sequence with the packing material of silica gel. The pentane fraction was found rich in 
alkanes and alkenes, in the benzene fraction polycyclic aromatic hydrocarbons (PAHs) were abundant, many phenol derivatives and some oxygen containing compounds were detected in dichloromethane and ethyl acetate fractions, and in the methanol fraction some polar compounds like sugars were more concentrated. In Das's work [14], the bio-oil from pyrolysis of cashew nut shell was successively eluted by hexane/ethyl acetate mixtures and chloroform/methanol mixtures in different ratio with the packing material of silica gel, and five monocomponents in five fractions were obtained, and each component has the common unique head- and -tail like structure with the phenolic group being at the head and a long linear chain forming the tail. Rutkowski et al. [15] separated a dehydrated bio-oil into fractions by eluting solvents of hexane, benzene, ether and methanol, with the packing material of neutral $\mathrm{Al}_{2} \mathrm{O}_{3}$. It was found that furan derivatives, phenol and its derivatives were the typical oxygen-containing compounds identified in ether and methanol fractions, and besides the methanol fraction was rich in C2-C10 carboxylic acids and glucose derivatives like levoglucosenone. In Xu's work [16], the neutral components in bio-oil obtained by solvent extraction was eluted by solvents of cyclohexane, benzene, and methanol in sequence with the column packing material of silica gel, and it was found that the main components in cyclohexane were paraffins, cycloparaffins, and PAHs; in benzene fraction, alkyl-aromatics, phenols, and a part of esters were abundant; the methanol fraction was rich in diisobutyl phthalate and di-n-butyl phthalate. Li et al. [17] fractionated the bio-oil free from water and asphaltene from pyrolysis of apricot pit and corncob by the same eluting solvents and the same column packing material as that in Xu's work, and the cyclohexane faction was found rich in PAHs; the components in benzene is more complex including acids, phenols, and five ring oxygen-containing compounds. Zhang et al. [18] eluted the volatile fraction of the bio-oil from sawdust, by solvents of n-heptane, benzene, and methanol with the column packing material of $\mathrm{Al}_{2} \mathrm{O}_{3}$, and it was found that the content of dimethyl-hydroxyphenol was the highest, and methyl furan, diethylphenol, phenethylol, phenyl acetic acid were also in high content.

In this paper, the column chromatographic separation of the original wood-tar (oil-phase of bio-oil from slow pyrolysis of wood) with the packing material of silica gel was tested, the components of the raw wood-tar was analyzed, and the effects of different eluting solvents were compared.

\section{Experimental method}

Silica gel (200-300 mesh) of $60 \mathrm{~g}$ was packed in a glass tube (inner-diameter $2.8 \mathrm{~cm}$ ), and then $30 \mathrm{~mL}$ wood-tar was added to the top of the column. Three solvents of cyclohexane, benzene (dichloromethane), methanol were used for elution in sequence, and the process was promoted by increasing column head pressure by balloon. The solvent in the eluting solution was firstly distilled away, and then the rest bottom solution was measured and analyzed by GC-MS. The corresponding conditions and measurements were listed in Table 1.

\section{Analysis of the raw wood-tar}

The composition analysis is the premise and guarantee for component separation. So herein the raw wood-tar was firstly analyzed by screened gas chromatogram column and optimized analytical conditions. The corresponding conditions are as follows: Varian 300 GC-MS; column of FFAP $25 \mathrm{~mm} \times 0.25 \mathrm{~mm} \times 0.2 \mu \mathrm{m}$; injector temperature $240^{\circ} \mathrm{C}$; EI temperature $250^{\circ} \mathrm{C}$; oven temperature starts from $35^{\circ} \mathrm{C}(1$ min), then increases to $50^{\circ} \mathrm{C}(1 \mathrm{~min})$ by $2^{\circ} \mathrm{C} / \mathrm{min}$, then increases to $80^{\circ} \mathrm{C}(1 \mathrm{~min})$ by $3^{\circ} \mathrm{C} / \mathrm{min}$, and then increases to $230^{\circ} \mathrm{C}(2 \mathrm{~min})$ by $4^{\circ} \mathrm{C} / \mathrm{min}$, and finally increases to $240^{\circ} \mathrm{C}$ (10 $\mathrm{min}$ ) by $4^{\circ} \mathrm{C} / \mathrm{min}$. The total ion chromatogram (TIC) of the raw wood-tar is illustrated in Figure 1.

It can be seen from Figure 1 that the components in wood-tar were complex, 191 peaks were found over 0.1 (area)\%, and 22 peaks were over 1 (area)\%. The components include almost all oxygen-containing compounds like phenols, alcohols, aldehydes, ketones, acids, ethers, esters, and some non-polar compounds like aromatics, paraffins, cycloalkanes, etc. Phenol derivatives were the most abundant components, occupying over 40(area)\%, and five ring oxygen-containing compounds were also in high content. Small alcohols and ketones mainly distributed in the retention time of 2-15 min; in the range of 15-35 min small acids and five ring oxygen-containing compounds were abundant; monophenols were in high content in 35-55 min; in 55-60 min diphenols were abundant; and in the range of 60-70 min scattered some fatty hydrocarbons and fatty acids. The main components in raw wood-tar and the corresponding area percentages were listed in Table 2.

\section{Results and discussion}

\subsection{Eluted by cyclohexane-benzene-methanol}

Wood-tar was eluted by cyclohexane-benzene-methanol

Table 1 Amount of the solvents and the bottom solution after distillation

\begin{tabular}{ccccc}
\hline Exp. & Solvent & $\begin{array}{c}\text { Volume of } \\
\text { solvent }(\mathrm{mL})\end{array}$ & $\begin{array}{c}\text { Mass of bot- } \\
\text { tom solution } \\
(\mathrm{g})\end{array}$ & $\begin{array}{c}\text { Volume of } \\
\text { bottom solu- } \\
\text { tion }(\mathrm{mL})\end{array}$ \\
\hline \multirow{3}{*}{ Exp.1\# } & Cyclohexane & 300 & 12.82 & 13.6 \\
& Benzene & 265 & 8.81 & 8.6 \\
& Methanol & 196.5 & 3.01 & 3.5 \\
& Cyclohexane & 304 & 12.48 & 13.1 \\
Exp.2\# & Dichloromethane & 262.5 & 13.13 & 11.7 \\
& Methanol & 243 & 7.36 & 6.4 \\
\hline
\end{tabular}




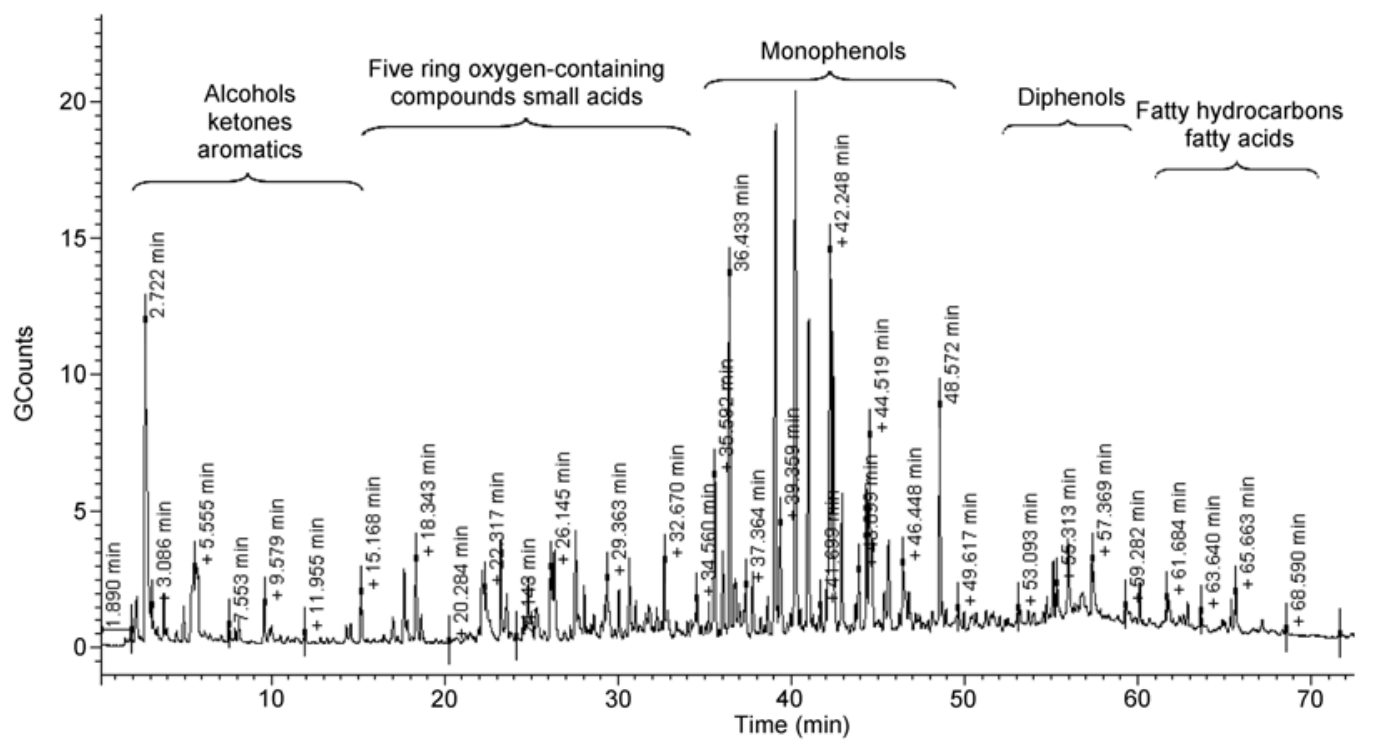

Figure 1 TIC of the raw wood-tar.

Table 2 Main components in the raw wood-tar and their area percentages

\begin{tabular}{|c|c|c|c|c|c|c|c|}
\hline No. & $\mathrm{RT}(\min )$ & Compound name & Area\% & No. & $\mathrm{RT}(\min )$ & Compound name & Area\% \\
\hline 1 & 2.722 & Methyl Alcohol & 4.069 & 22 & 40.991 & Phenol, 4-ethyl-2-methoxy- & 2.795 \\
\hline 2 & 5.555 & Water & 2.505 & 23 & 42.248 & $\begin{array}{c}\text { Mixture of 4-methyl-phenol and } \\
\text { 2,4-dimethyl-phenol }\end{array}$ & 4.206 \\
\hline 3 & 17.657 & 2-Cyclopenten-1-one & 0.602 & 24 & 42.398 & Phenol, 3-methyl- & 2.742 \\
\hline 4 & 18.343 & 2-Cyclopenten-1-one, 2-methyl- & 0.774 & 25 & 42.928 & Phenol, 2-methoxy-4-propyl- & 1.126 \\
\hline 5 & 22.137 & Acetic acid & 1.843 & 26 & 43.899 & Phenol, 2,3-dimethyl- & 0.727 \\
\hline 6 & 23.235 & Furfural & 0.796 & 27 & 44.325 & Phenol,2-ethyl-5-methyl- & 1.155 \\
\hline 7 & 24.831 & Ethanone, 1-(2-furanyl)- & 0.595 & 28 & 44.519 & Phenol, 3,5-dimethyl- & 1.672 \\
\hline 8 & 25.299 & 2-Cyclopenten-1-one, 3-methyl- & 0.543 & 29 & 44.657 & Phenol, 3-ethyl- & 0.768 \\
\hline 9 & 26.145 & Butanoic acid, 2-oxo- & 1.045 & 30 & 45.612 & Phenol, 3,4-dimethyl- & 1.115 \\
\hline 10 & 26.333 & 2-Cyclopenten-1-one, 2,3-dimethyl- & 0.971 & 31 & 46.448 & Phenol, 2-methoxy-4-(1-propenyl)- or Eugenol & 1.179 \\
\hline 11 & 27.556 & 2-Furancarboxaldehyde, 5-methyl- & 0.934 & 32 & 46.788 & Phenol, 2,6- dimethoxy- & 0.58 \\
\hline 12 & 29.363 & Pentanoic acid & 0.793 & 33 & 48.572 & Phenol, 2-methoxy-4-(1-propenyl)- or Eugenol & 1.836 \\
\hline 13 & 30.651 & 2-Furanmethanol & 0.556 & 34 & 49.617 & 1,4:3,6-Dianhydro-.alpha.-d-glucopyranos & 0.824 \\
\hline 14 & 32.67 & Naphthalene & 0.585 & 35 & 55.117 & 2-Propanone, 1-(4-hydroxy-3-methoxyphenyl)- & 0.675 \\
\hline 15 & 35.592 & 1,2-Cyclopentanedione, 3-methyl- & 1.554 & 36 & 55.313 & 1,2-Benzenediol, 3-methyl- & 1.04 \\
\hline 16 & 36.048 & Naphthalene, 2-methyl- & 0.685 & 37 & 55.982 & 1,2-Benzenediol & 1.42 \\
\hline 17 & 36.433 & Phenol, 2-methoxy- & 2.962 & 38 & 56.817 & 2,5-Dimethylhydroquinone & 1.022 \\
\hline 18 & 37.772 & Phenol, 2,6-dimethyl- & 0.558 & 39 & 57.369 & 1,2-Benzenediol, 4-methyl- & 1.523 \\
\hline 19 & 39.1 & Phenol, 2-methoxy-4-methyl- & 4.642 & 40 & 59.282 & 3-Allyl-6-methoxyphenol & 0.547 \\
\hline 20 & 39.359 & Unknown & 1.148 & 41 & 65.663 & Oleic acid & 0.559 \\
\hline 21 & 40.232 & Phenol mixed with 2-methyl-phenol & 5.22 & & & Sum & 60.891 \\
\hline
\end{tabular}

with the packing material of silica gel. After the solvent in the eluting solution was distilled away, the bottom solution was analyzed. The total ion chromatograms of the bottom solutions were shown in Figures 2-4, and the area percentages of the main components were listed in Tables $3-5$.

It can be seen from Figure 2 and Table 3 that monophenols were the most abundant components in the cyclohexane fraction; other types of compounds were rather little. Among the monophenols, alkoxyl-monophenols were more distinct than alkyl-monophenols. For instance, the area per- centages of 4-methyl-2-methoxy-phenol, 4-ethyl-2-methoxyphenol, and 2-methoxy-phenol were all over 5(area)\%. Alkyl-monophenols like 2-methyl-phenol and 2,4-dimethylphenol were also in higher content, but less than those of alkoxyl-monophenols.

Figure 3 and Table 4 showed that monophenols were still the most abundant component in benzene, while the composition of the monophenols was different to that in cyclohexane. The content of alkyl-monophenols increased obviously, higher than that of alkoxyl-monophenols. For instance, the content of phenol, 4-methyl-phenol, and 3-me- 


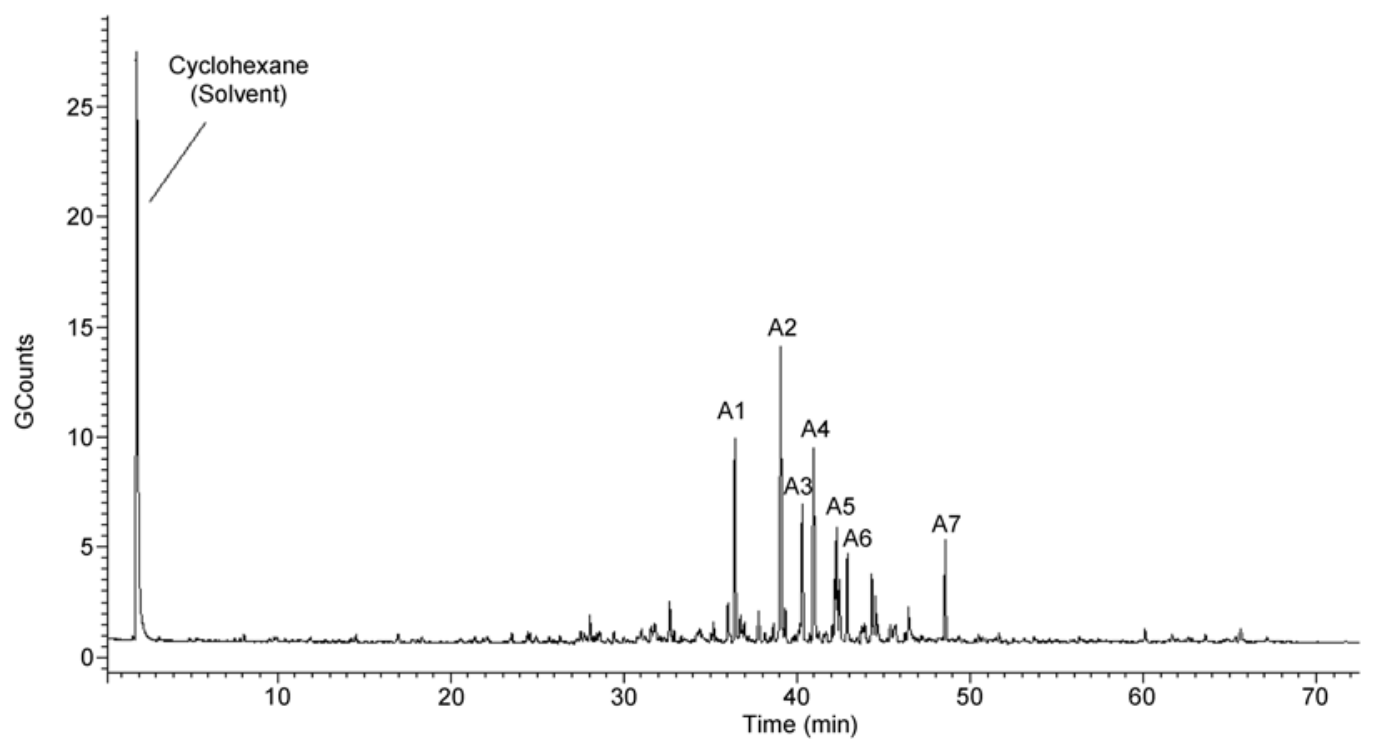

Figure 2 TIC of the distilled cyclohexane solution.

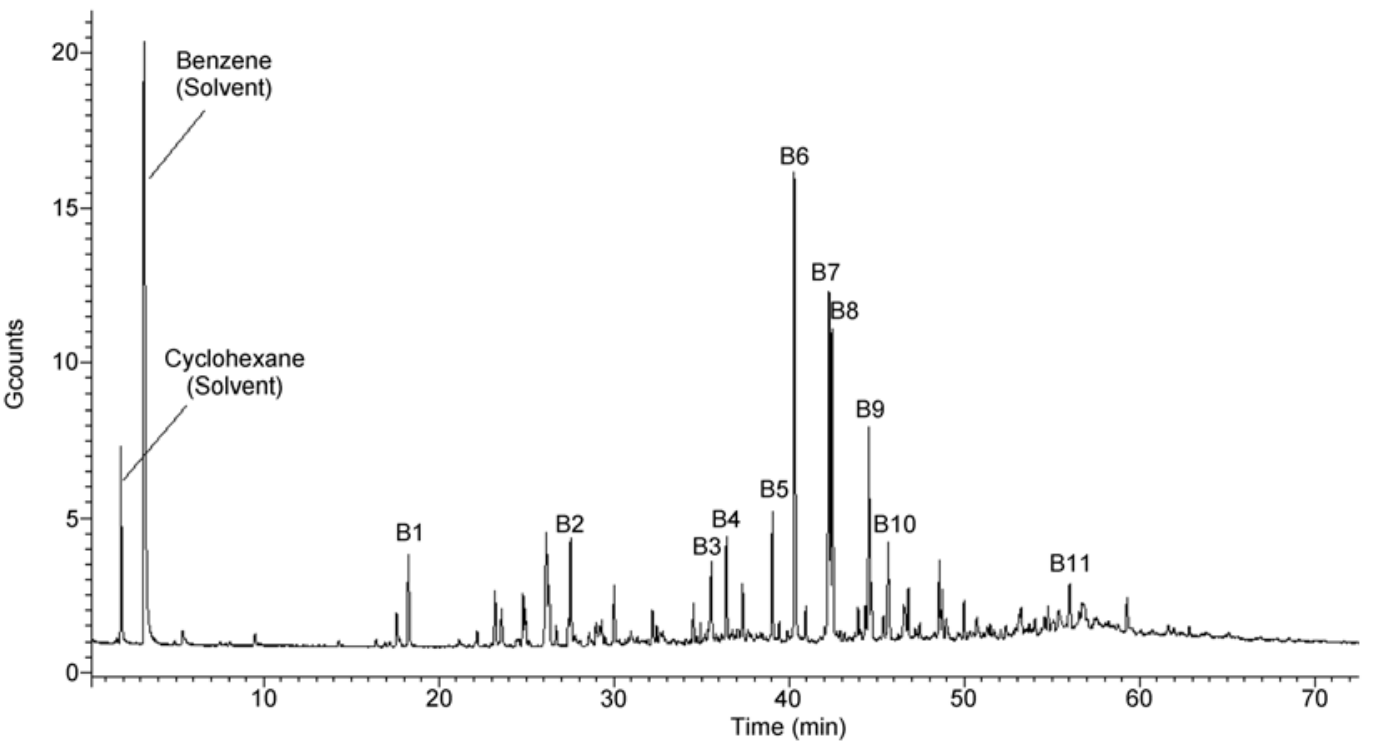

Figure 3 TIC of the distilled benzene solution.

thyl-phenol was higher than that of the top abundant alkoxyl-monophenol of 2-methoxy-4-methyl-phenol. The content of five ring oxygen-containing compounds also increased a lot, and the main representative components were 5-methyl-2-furancarboxaldehyde, 2-hydroxy-3-methyl-2cyclopenten-1-one, and 2-methyl-2-cyclopenten-1-one.

It can be seen from Figure 4 and Table 5 that the types of the components were diverse and the diphenols were relatively more abundant. For instance, the compound of 4-methyl-1,2-benzenediol was the richest component. The five ring oxygen-containing compounds like 3-methyl-1,2cyclopentanedione and 3-methyl-2-cyclopenten-1-one were also in a relatively higher content. The content of monophenols including both alkyl- and alkoxyl- monophenols decreased generally, while it was interesting that the monophenol compound of 1-(4-hydroxy-3-methoxyphenyl)-2propanone was in a rather high content.

It can be seen from the above results that the wood-tar eluted by cyclohexane-benzene-methanol with the packing material of silica gel can be separated into three feature distinct fractions. In general, the alkoxyl- monophenols were most abundant in cyclohexane; the alkyl-monophenols and five ring oxygen-containing compounds were rich in benzene; in methanol, the components were diverse and the content of diphenols was relatively higher. In the viewpoint of application or post processing of the three fractions, the process for obtaining the cyclohexane fraction may be referential to the concentration of alkoxyl-monophenols, or to 


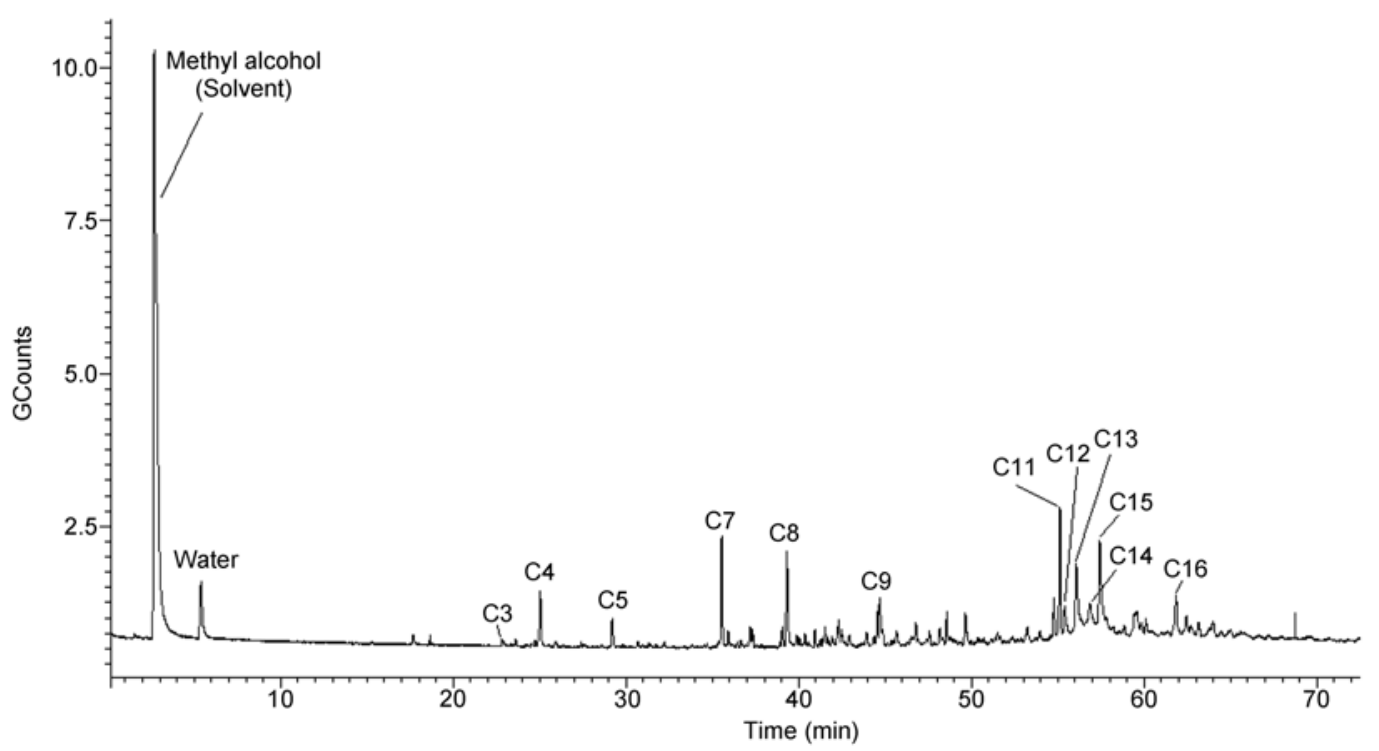

Figure 4 TIC of the distilled methanol solution after being eluted by benzene.

Table 3 Main components in distilled cyclohexane system

\begin{tabular}{lccc}
\hline No. & RT $(\mathrm{min})$ & Compound name & Area\% \\
\hline A1 & 36.423 & Phenol, 2-methoxy- & 5.39 \\
A2 & 39.068 & Phenol, 2-methoxy-4-methyl- & 9.023 \\
A3 & 40.311 & Phenol, 2-methyl- & 3.903 \\
A4 & 40.966 & Phenol, 4-ethyl-2-methoxy- & 5.766 \\
A5 & 42.289 & Phenol, 2,4-dimethyl- & 3.043 \\
A6 & 42.917 & Phenol, 2-methoxy-4-propyl- & 2.42 \\
A7 & 48.568 & Phenol, 2-methoxy-4-(1-propenyl)-, (E)- & 2.48 \\
\hline
\end{tabular}

Table 4 Main components in distilled benzene and dichloromethane systems

\begin{tabular}{|c|c|c|c|c|c|c|}
\hline \multirow{2}{*}{ No. } & \multirow{2}{*}{$\mathrm{RT}(\min )$} & \multirow{2}{*}{ Compound name } & \multicolumn{2}{|c|}{ Exp.1\# } & \multicolumn{2}{|c|}{ Exp.2\# } \\
\hline & & & Area\% & Sum & Area\% & Sum \\
\hline B1 & 18.278 & 2-Cyclopenten-1-one, 2-methyl- & 1.702 & \multirow{3}{*}{5.355} & 1.557 & \multirow{3}{*}{5.529} \\
\hline B2 & 27.543 & 2-Furancarboxaldehyde, 5-methyl- & 1.872 & & 1.615 & \\
\hline B3 & 35.549 & 1,2-Cyclopentanedione, 3-methyl- or 2-hydroxy-3-methyl-2-cyclopenten-1-one & 1.781 & & 2.357 & \\
\hline B4 & 36.42 & Phenol, 2-methoxy- & 1.62 & \multirow{2}{*}{3.511} & 1.795 & \multirow{2}{*}{3.782} \\
\hline B5 & 39.039 & Phenol, 2-methoxy-4-methyl- & 1.891 & & 1.987 & \\
\hline B6 & 40.29 & Phenol mixed with 2-methyl-phenol & 8.914 & & 8.684 & \multirow{4}{*}{26.312} \\
\hline B7 & 42.272 & Phenol, 4-methyl- & 7.249 & \multirow{3}{*}{27.423} & 7.069 & \\
\hline B8 & 42.438 & Phenol, 3-methyl- & 5.777 & & 5.418 & \\
\hline B9 & 44.537 & Phenol, 3,5-dimethyl- & 3.514 & & 3.359 & \\
\hline B 10 & 45.64 & Phenol, 3,4-dimethyl- & 1.969 & & 1.782 & \\
\hline \multirow[t]{2}{*}{ B11 } & 55.12 & 2-Propanone, 1-(4-hydroxy-3-methoxyphenyl)- & $0.233^{\mathrm{a})}$ & & 1.473 & \\
\hline & & Summary & 36.522 & & 37.096 & \\
\hline
\end{tabular}

a) Non-major component, just for comparison.

separation between alkoxyl- and alkyl- monophenols; the abundant alkyl-monophenols and five ring oxygencontaining compounds in benzene can be further separated by solvent extraction due to their different acidities; the methanol fraction can be a source for more concentrated diphenols, and the other components can be more conveniently concentrated by post treatments like distillation and/or solvent extraction, since the diverse components were more different in properties. It is worth mentioning that the structure unique compound of 1-(4-hydroxy-3methoxyphenyl)-2-propanone in high content in methanol, makes the further concentration of this component more easily, bringing an opportunity for its fine extraction.

Silica gel has a stronger polarity due to the rich hydroxyl 
Table 5 Main components in distilled methanol systems

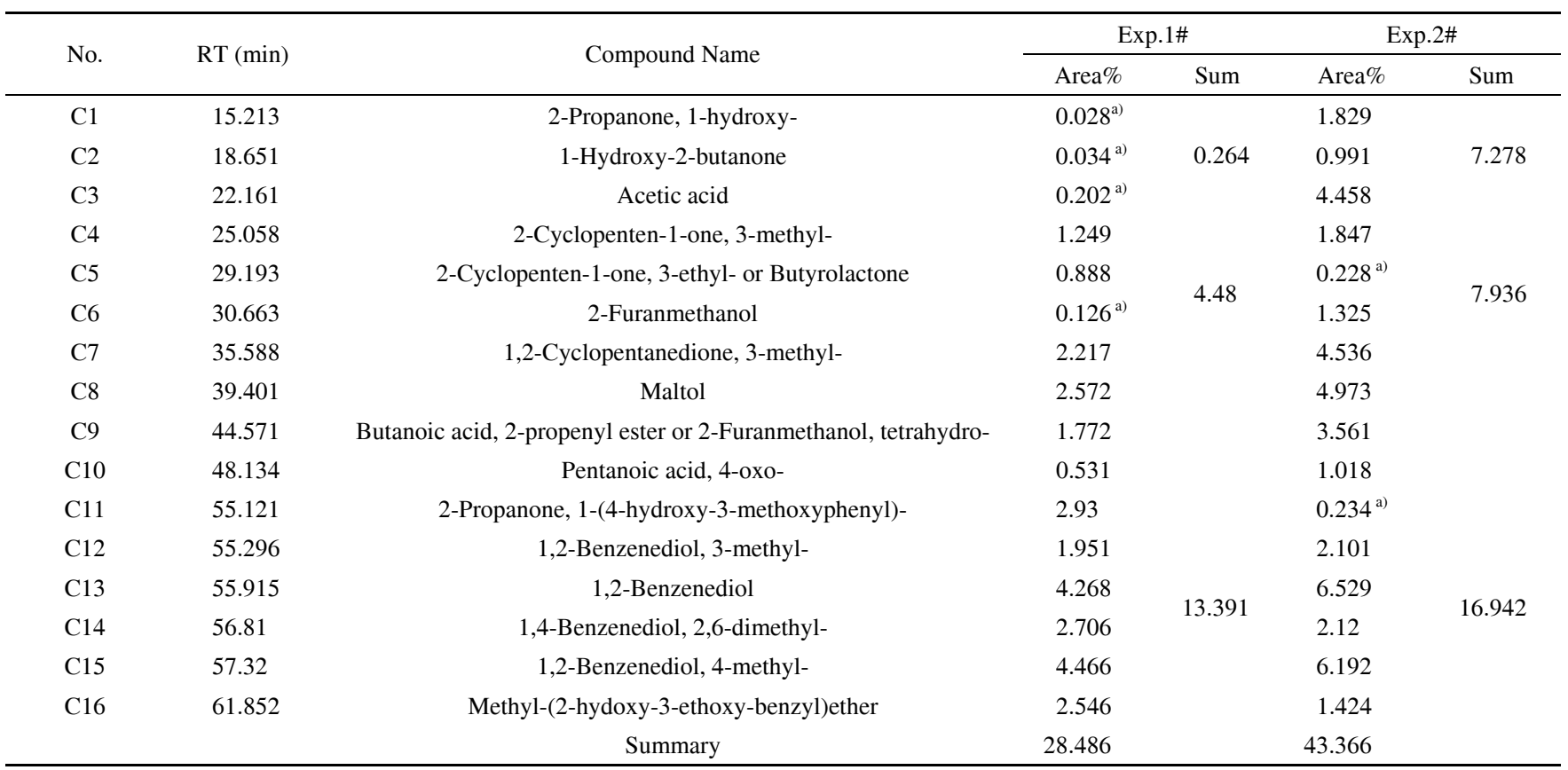

a) Non-major component, just for comparison.

group on the surface. When the wood-tar was eluted by non-polar solvent of cyclohexane, the component with stronger polar bond has a more intense force with silica gel and harder to be eluted off the column compared to that with weaker polar bond. The different eluting effect by cyclohexane between alkoxyl- and alkyl-monophenols is related to the difference between their molecular structures. Alkoxyl and alkyl group are both electron-donating groups. The repulsive effect of the electron-donating group makes the electron density on the benzene ring increase, and makes the polarity of the phenolic hydroxyl bond $\delta_{\mathrm{O}-\mathrm{H}}$ on the ortho-position and para-position decrease through the alternative polarization on the benzene ring by conjugative effect[19]. Alkoxyl group has a stronger electron-donating effect than alkyl group, so the force between silica gel and the phenolic hydroxyl bond of alkoxyl-monophenol is weaker than that of alkyl-monophenol, and therefore the alkoxyl-monophenols are easier to be eluted by cyclohexane than alkyl-monophenols. When the solvent of benzene with higher solubility and higher polarity than cyclohexane is applied, besides the remaining alkoxyl- and alkyl-monophenols, some stronger polar five ring oxygen-containing compounds were also eluted away by benzene. When the solvent of methanol with even higher polarity was applied, more components in even higher polarities including dihydric compounds like diphenols, multi oxygen containing compound like 1-(4-hydroxy-3-methoxyphenyl)-2-propanone and Maltol, and some remaining five ring oxygen-containing compounds were eluted by methanol. So the wood-tar can be separated to three feature-distinct fractions when eluted by the solvents of cyclohexane-benzene-methanol with the packing material of silica gel.

\subsection{Eluted by cyclohexane-dichloromethane-methanol}

In the above case (Exp.1), the solvent of benzene is a toxic agent with a relatively higher boiling point $\left(80^{\circ} \mathrm{C}\right)$, which is disadvantageous for the operating security and the stableness of those heat sensitive components, so herein the solvent of dichloromethane with slight toxicity and low boiling point $\left(40^{\circ} \mathrm{C}\right)$ in displacement of benzene was tested with the same packing material of silica gel. The total ion chromatograms of the distilled dichloromethane and methanol solutions were shown in Figures 5 and 6, and the compositions of the two systems were listed in Tables 4 and 5 respectively, together with the former results obtained from Exp.1 for convenient comparisons.

It can be seen from Figure 5 and Table 4 that the compositions of the dichloromethane and benzene systems were very similar. In dichloromethane fraction, the alkoxyl-monophenols and five ring oxygen-containing compounds were still in high content and still less than the most abundant components of alkyl-monophenols as that in benzene, and phenol and 4-methyl-phenol were still the top two abundant compounds. While the content of alkyl-monophenols in dichloromethane was reduced a little bit, and the compound of 1-(4-hydroxy-3-methoxyphenyl)-2-propanone increased obviously compared with that in benzene. It may be because the polarity of dichloromethane is higher than that of benzene, and easier to elute away more polar components.

It can be seen from Figure 6 and Table 5 that similar to 


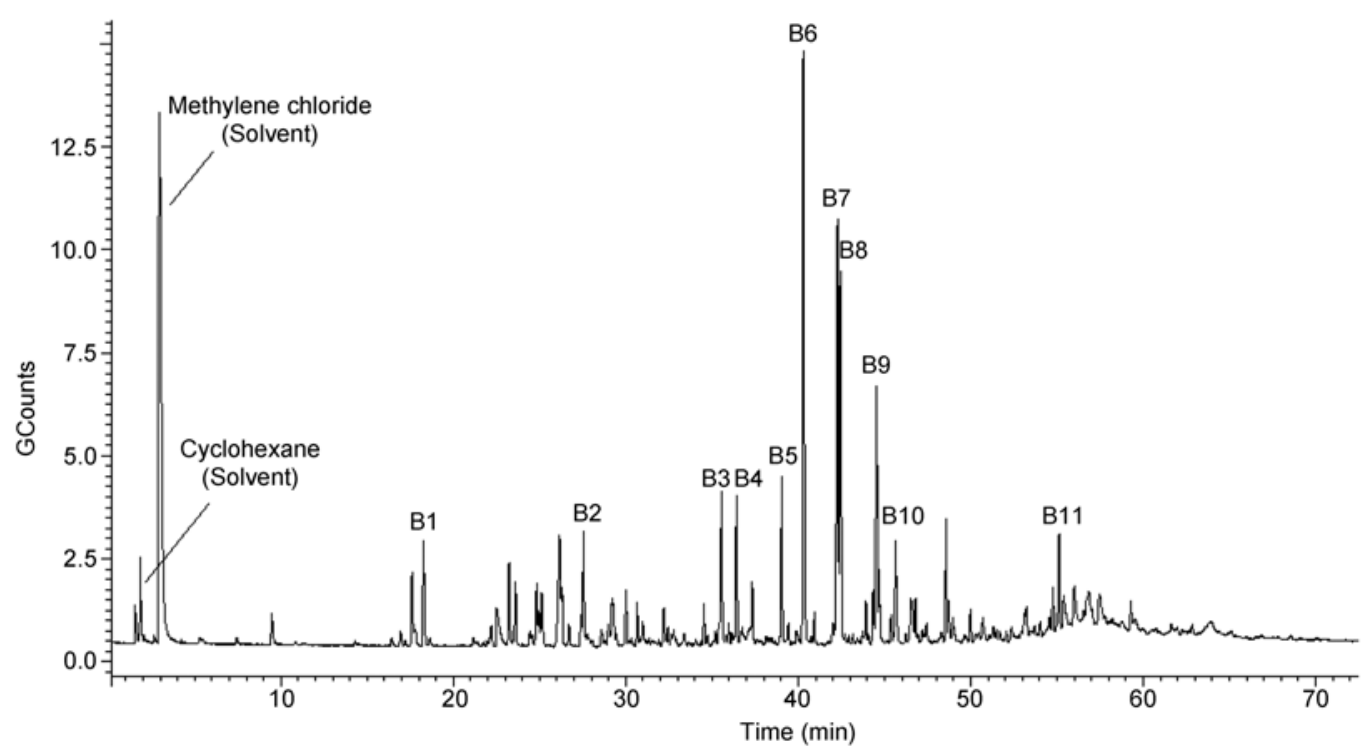

Figure 5 TIC of the distilled dichloromethane solution.

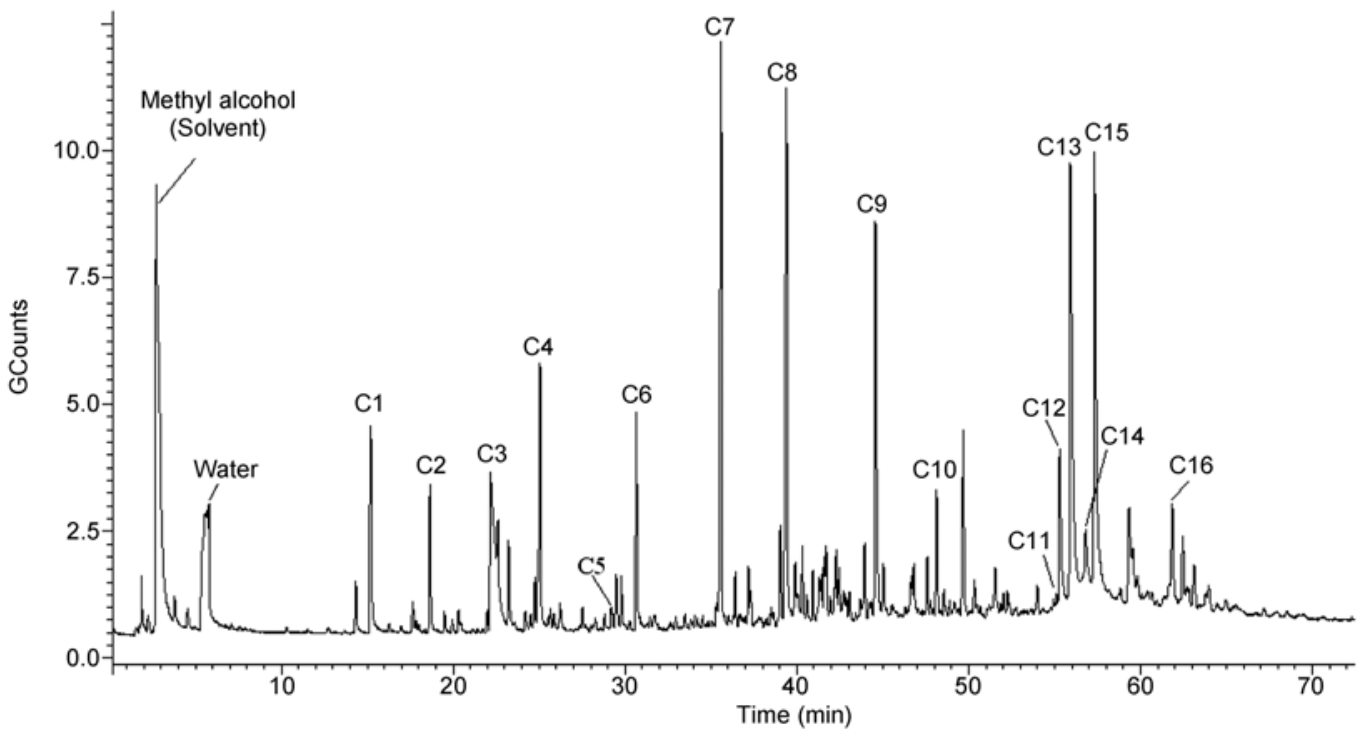

Figure 6 TIC of the distilled methanol solution after being eluted by dichloromethane.

the case after benzene elution, the components in methanol after being eluted by dichloromethane were also very diverse, diphenols were relatively higher too, and the most abundant diphenols were still 1,2-benzenediol and 4-methyl-1,2-benzenediol. But the five ring oxygen-containing compounds and some small polar molecules like 3-methyl-1,2-cyclopentanedione, acetic acid, and 1-hydroxy-2-propanone were more concentrated, and on the contrary the content of 1-(4-hydroxy-3-methoxyphenyl)-2-propanone was distinctly reduced, compared with that after being eluted by benzene.

The increase of the small polar molecules and five ring oxygen-containing compounds in methanol after being eluted by dichloromethane should be related to the difference of the polarity between dichloromethane and benzene.
The polarity of dichloromethane is a little bit higher than that of benzene but still in low level generally. Most of the of 1-(4-hydroxy-3-methoxyphenyl)-2-propanone can be eluted off the column by dichloromethane, while the more polar compounds like acids are still hard to be completely eluted away, but to the near end district of the column, and it makes these components more easily to be eluted by methanol, and therefore makes their content higher in the methanol fraction.

\section{Conclusions}

The column chromatographic fractionation of wood-tar was 
investigated, by two series of eluants of cyclohexane-benzene-methanol and cyclohexane-dichloromethane-methanol, with the packing material of silica gel. It was found that the content of alkoxyl-monophenols was the highest in cyclohexane; the components of alkyl-monophenols and five ring oxygen-containing compounds were abundant in benzene and in dichloromethane fractions similarly, while the content of alkyl-monophenols in dichloromethane reduced a little bit compared with that in benzene system; in methanol the components were diverse and diphenols were in higher content relatively, and comparatively some small polar molecules and five ring oxygen-containing compounds were more abundant in the methanol fraction after being eluted by dichloromethane, and the content of 1-(4-hydroxy-3methoxyphenyl)-2-propanone was higher in the methanol solution after being eluted by benzene.

The higher content of alkoxyl-monophenols in cyclohexane indicates a referable approach for the separation of alkoxyl-monophenols and alkyl-monophenols. The methanol fraction can be a source for more concentrated diphenols, and the other components inside can be more conveniently concentrated by post treatments like distillation and/or solvent extraction too. The compound of 1-(4-hydroxy-3-methoxyphenyl)-2-propanone in high content in methanol after being eluted by benzene makes the concentrating of this component more easily, bringing an opportunity for its fine extraction.

This work was supported by the National Basic Research Program of China (2004CB719704), and the National Natural Science Foundation of China (0202017127).

1 Mohan D, Pittman C U, Steele P H. Pyrolysis of wood/biomass for bio-oil: A critical review. Energ Fuel, 2006, 20: 848-889

2 Bridgwater A V. Renewable fuels and chemicals by thermal processing of biomass. Chem Eng J, 2003, 91: 87-102

3 Karagöz S, Bhaskar T, Muto A, et al. Low-temperature catalytic hydrothermal treatment of wood biomass: Analysis of liquid products. Chem Eng J, 2005, 108: 127-137

4 Karagöz S, Bhaskar T, Muto A, et al. Comparative studies of oil compositions produced from sawdust, rice husk, lignin and cellulose by hydrothermal treatment. Fuel, 2005, 84: 875-884

5 American Can Company. Fractionation of oil obtained by pyrolysis of lignocellulosic materials to recover a phenolic fraction for use in making phenol-formaldehyde resins. US Patent, US4209647, 1980-06-24

6 Midwest Research Institute. Process for fractionating fast-pyrolysis oils, and products derived therefrom. US Patent, US4942269, 1990-07-17

$7 \mathrm{Xu} \mathrm{B} \mathrm{J,} \mathrm{Lu} \mathrm{N,} \mathrm{Li} \mathrm{J} \mathrm{S,} \mathrm{et} \mathrm{al.} \mathrm{Experimental} \mathrm{research} \mathrm{on} \mathrm{the} \mathrm{bio-oil} \mathrm{de-}$ rived from biomass pyrolysis liquefaction (in Chinese). Trans Chin Soc Agric Engin, 1999, 15: 177-181

8 Pütün A E, Özcan A, Pütün E. Pyrolysis of hazelnut shells in a fixed-bed tubular reactor: Yields and structural analysis of bio-oil. J Anal Appl Pyroly, 1999, 52: 33-49

9 Boucher M E, Chaala A, Roy C. Bio-oils obtained by vacuum pyrolysis of softwood bark as a liquid fuel for gas turbines. Part I: Properties of bio-oil and its blends with methanol and a pyrolytic aqueous phase. Biomass Bioenerg, 2000, 19: 337-350

10 Adjaye J D, Sharma R K, Bakhshi N N. Characterisation and stability analysis of wood-derived bio-oil. Fuel Process Technol, 1992, 31: 241-256

11 Murwanashyaka J N, Pakdel H, Roy C. Seperation of syringol from birch wood-derived vacuum pyrolysis oil. Sep Purif Technol, 2001, 24: $155-165$

12 Guo Z G, Wang S R, Zhu Y Y, et al. Separation of acid compounds for refining biomass pyrolysis oil (in Chinese). J Fuel Chem Technol, 2009, 37: 49-52

13 Ba T, Chaala A, Garcia-Perez M, et al. Colloidal properties of bio-oils obtained by vacuum pyrolysis of softwood bark storage stability. Energ Fuel, 2004, 18: 188-201

14 Das P, Sreelatha T, Ganesh A. Bio oil from pyrolysis of cashew nut shell-characterisation and related properties. Biomass Bioenerg, 2004, 27: 265-275

15 Rutkowski P. Influence of zinc chloride addition on the chemical structure of bio-oil obtained during co-pyrolysis of wood/synthetic polymer blends. Waste Manage, 2009, 29: 2983-2993

16 Xu S P, Liu J, Li S G, et al. Solvent extraction-column chromatographic separation of bio-oil from fast pyrolysis of apricot stone and manufacture technology (in Chinese). J Dalian Univer Technol, 2005, 45: 505-510

17 Li S G, Xu S P, Lu Q H, et al. Column chromatographic separation of bio-oil from fast pyrolysis of biomass (in Chinese). Acta Energ Sol Sin, 2005, 26: 549-555

18 Zhang S P, Yan Y J, Ren Z W, et al. Analysis of liquid product obtained by the fast pyrolysis of biomass (in Chinese). J East China Univer Sci Tech, 2001, 27: 666-668

19 Guo H M. Explanation on the effect of position and type of substituent group on the chemical properties of aromatic compound by electronic effect regulations (in Chinese). Univer Chem, 2008, 23: 54-57

Open Access This article is distributed under the terms of the Creative Commons Attribution License which permits any use, distribution, and reproduction in any medium, provided the original author(s) and source are credited. 